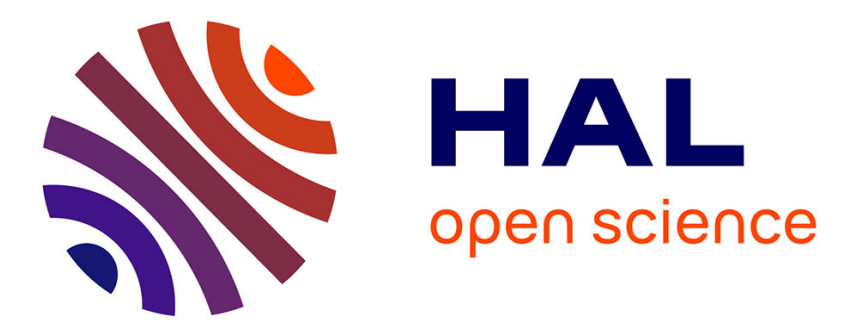

\title{
Étude des perceptions de l'environnement de travail au cours du poste chez des travailleurs postés : intérêt d'une évaluation subjective et temporellement située de la charge de travail
}

Nadine Cascino, Claudine Mélan, Edith Galy

\section{To cite this version:}

Nadine Cascino, Claudine Mélan, Edith Galy. Étude des perceptions de l'environnement de travail au cours du poste chez des travailleurs postés : intérêt d'une évaluation subjective et temporellement située de la charge de travail. Le travail humain, 2016, 79 (2), pp.97-124. 10.3917/th.792.0097. hal-01461491

\section{HAL Id: hal-01461491 \\ https://hal-amu.archives-ouvertes.fr/hal-01461491}

Submitted on 16 Feb 2017

HAL is a multi-disciplinary open access archive for the deposit and dissemination of scientific research documents, whether they are published or not. The documents may come from teaching and research institutions in France or abroad, or from public or private research centers.
L'archive ouverte pluridisciplinaire HAL, est destinée au dépôt et à la diffusion de documents scientifiques de niveau recherche, publiés ou non, émanant des établissements d'enseignement et de recherche français ou étrangers, des laboratoires publics ou privés. 
RECHERCHES EMPIRIQUES EMPIRICAL STUDIES

\title{
ÉTUDE DES PERCEPTIONS DE L'ENVIRONNEMENT DE TRAVAIL AU COURS DU POSTE CHEZ DES TRAVAILLEURS POSTÉS : INTÉRÊT D'UNE ÉVALUATION SUBJECTIVE ET TEMPORELLEMENT SITUÉE DE LA CHARGE DE TRAVAIL
}

\author{
Par Nadine Cascino ${ }^{1}$, Claudine MÉlan ${ }^{2}$, Édith Galy ${ }^{3}$
}

RÉSUMÉ

Pour mieux comprendre les effets du travail en horaires postés, nous avons étudié les variations des perceptions de l'environnement de travail selon le poste travaillé et la phase du poste. Le questionnaire construit, en référence au fob Content Questionnaire, mesure les perceptions de demandes, contrôle et soutien social et deux dimensions supplémentaires : les ressources techniques et humaines et les interférences travail-hors travail. Il a été renseigné à trois reprises (une heure après le début, en milieu et une heure avant la fin du poste) pour chaque poste occupé (matin, après-midi, nuit) par 38 personnels de soins (86 observations). Les résultats montrent des demandes psychologiques globalement plus élevées en début et milieu de poste et des demandes physiques plus élevées en fin de poste. Lors du poste de nuit, les personnels perçoivent davantage d'autonomie et moins de soutien des collègues. Des interactions entre le poste et la phase du poste indiquent une augmentation des ressources sur le poste du matin, une diminution l'après-midi et des valeurs stables la nuit. Pris ensemble, ces résultats indiquent que, si le poste de nuit paraît le moins exposé à la tension professionnelle, celui du matin donne lieu à des perceptions de stress positif en milieu de poste et de faible tension en fin de poste tandis que la tension professionnelle augmente tout au long du poste de l'après-midi. Ils soulignent l'importance d'une évaluation de la charge de travail selon le poste et le moment du poste chez des personnels de soins et de considérer différentes ressources en accord avec le modèle "job demandsresources" (Demerouti et al., 2001). Bien qu'ils ne puissent être généralisés à d'autres situations de travail et doivent être discutés à la lumière de l'activité de

1. CLLE-LTC, Cognition, langage, langue et ergonomie-laboratoire travail et cognition, université de Toulouse Jean Jaurès, 5 allées Antonio Machado, 31058 Toulouse cedex 9 cascino@univ-tlse2.fr

2. CLLE-LTC, Cognition, langage, langue et ergonomie-laboratoire travail et cognition, université de Toulouse Jean Jaurès, 5 allées Antonio Machado, 31058 Toulouse cedex 9 cmelan@univ-tlse2.fr

3. PsyCLé, Centre de recherche en psychologie de la connaissance, du langage et de l'émotion, université d'Aix-en-Provence, 29 avenue Robert Schuman, 13621 Aix-en-Provence cedex 01 - edith.marie@univ-amu.fr 
travail, la méthodologie développée apporte des données complémentaires à celles obtenues avec une approche plus globale.

Mots-clés : demandes-contrôle-soutien social, stress, charge mentale, travail posté, moment du poste, personnel de soins.

\section{SUMMARY}

VARIABILITY OF JOB PERCEPTIONS ACROSS THE SHIFT IN SHIFT-WORKERS: ARGUMENTS FOR A SUBJECTIVE AND TEMPORALLY LOCATED ASSESSMENT OF WORKLOAD

In France, $17 \%$ of employees are currently concerned by shift-work. For a better understanding of the psychological effects of shift-work, we evaluated the extent to which perceptions of the job environment vary according to the shift worked, and to the shift-phase. A self-administered questionnaire was elaborated to measure job perceptions of 14 caregivers and 24 nurses (86 observations). In reference to the fob Content Questionnaire (FCQ, Karasek et al., 1998), the questionnaire addressed psychological and physical demands, control, supervisor and co-worker social support, and two additional dimensions: availability of technical and human resources and work-family interferences. Each participant completed the questionnaire three times (one hour after shift-beginning, in the middle of the shift, one hour before shift-end) on each shift worked (morningshift, afternoon-shift and night-shift). Results indicated overall higher psychological demands at the beginning and in the middle of the shift than at the shift-end, and higher physical demands at the shift-end. Further, on the nightshift compared to the morning-and afternoon-shifts, the perception of autonomy was higher, while social support by co-workers was lower. These main effects were qualified by an interaction between shift and shift-phase: co-worker support and availability of technical and human resources increased across the morning-shift, decreased across the afternoon-shift and remained stable during the night-shift. Together with the results concerning perceived job demands, these results indicate that during the night shift, participants were less exposed to job strain, especially in the middle of the shift. During the morning-shift, job perceptions indicated positive stress in the middle of the shift and low tension at the shift-end, whereas during the afternoon-shift the professional tension increased throughout the shift. Thus, a differential assessment of job perceptions according to the shift and shiftphase is important in order to assess perceived workload that would be determined by both psychological and physical job demands in healthcare staff. Our findings also highlight the importance of introducing the dimension of technical and human resources in addition to social support and control, in agreement with the "job demands-resources" model (FD-R; Demerouti et al., 2001). Even though these results may not be extended to other job situations, and need to be interpreted in light of task organization across the 24-h day in this job-situation, the methodology provides complementary information to the more general approach of job perceptions.

Keywords: "demand-control-social support" model, stress, shift-work, workload, shift-phase, healthcare workers. 


\section{INTRODUCTION}

La question posée dans cette étude est de savoir si, chez des professionnels de soins travaillant en horaires postés, les perceptions des caractéristiques de l'environnement de travail varient selon le poste occupé et au cours du poste de travail reflétant ainsi des variations de la charge de travail.

\section{I.1 À PROPOS DES PERCEPTIONS DE L'ENVIRONNEMENT DE TRAVAIL}

De nombreux facteurs ont été décrits comme pouvant contribuer à la perception d'un environnement de travail comme étant stressant, et en particulier les demandes liées au travail, les conditions de l'environnement, l'organisation du travail et les relations humaines (Costa, 1995). Selon le modèle "demande-contrôle-soutien social" (JDC-S ; Karasek, 1979 ; Theorell \& Karasek, 1996), des niveaux élevés de stress professionnel et des troubles consécutifs de la santé s'observent plus souvent dans des situations de travail caractérisées par des demandes psychologiques élevées, associées à un faible contrôle et à un soutien professionnel peu adapté ("situation professionnelle à forte tension "). D'un autre côté, des situations professionnelles caractérisées non seulement par des demandes élevées mais également par une autonomie élevée et un soutien professionnel adapté favorisent un comportement actif qui stimule l'apprentissage, la motivation et la productivité.

Le modèle "job demands-resources" (JD-R; Demerouti, Bakker, Nachreiner, \& Schaufeli, 2001) insiste sur le fait que chaque situation professionnelle présente des caractéristiques spécifiques qui peuvent être classées dans la catégorie des demandes ou des ressources (Van Emmerik, Bakker, \& Euwena, 2009). Les ressources constituent des aspects permettant d'atteindre les buts professionnels, de réduire les demandes et de stimuler le développement personnel (Shaufeli \& Bakker, 2004). Si ce modèle réaffirme le rôle de l'autonomie et du soutien social des collègues et supérieurs, d'autres ressources comme le feedback sur la performance sont prises en considération (Bakker, Demerouti, \& Schaufeli, 2003 ; Bakker, Demerouti, \& Sanz-Vergel, 2014). Dans cette perspective, il était essentiel pour nous d'élargir l'évaluation des ressources à des aspects plus directement liés à la réalisation des tâches au-delà des demandes physiques et psychologiques liées à l'activité de travail. Nous avons ainsi défini une catégorie "ressources techniques et humaines" pour évaluer d'une part, les outils disponibles (équipements adaptés, disponibilités d'informations nécessaires à la réalisation de la tâche) et d'autre part, les modalités d'organisation du travail en équipe telles les possibilités de coordination et l'existence de lieux et temps d'échanges (Mélan \& Cascino, 2014 ; Mélan, Cascino, \& Galy, 2014).

Par ailleurs, nombre de travaux ont décrit les conflits entre le travail et la vie hors travail (Gadbois, 1975 ; Gadbois, 2004 ; Rutenfranz, Colquhoun, Knauth, \& Ghata, 1977 ; Greenhaus \& Beutell, 1985 ; Demerouti, Geurts, Bakker, \& Euwena, 2004 ; Estryn-Behar \& Van der 
Hejden, 2012). Greenhaus et Beutell (1985) soulignent notamment que la participation dans un de ces domaines rend difficile de satisfaire les exigences de l'autre. Si la plupart du temps ces travaux examinent la façon dont le travail impacte la vie personnelle, notamment familiale, et le bien-être général, ils sous-tendent que les relations entre ces deux champs peuvent être réciproques. Ainsi, la satisfaction des rôles familiaux peut induire, au cours de l'activité de travail, des préoccupations spécifiques (démarches personnelles à organiser, inquiétude liée à l'état de santé d'un enfant,...) pouvant interférer avec la réalisation de l'activité de travail. Dans cette perspective, nous avons intégré une dimension " interférences du hors travail dans le travail " pour mieux appréhender les diverses demandes que les personnels sont susceptibles d'avoir à gérer durant le temps de travail (Mélan, Cascino, Galy, \& Barthe, 2012) afin de mieux saisir l'ensemble des exigences auxquelles les personnels doivent répondre au cours de leur travail.

\section{I.2. EFFETS DES HORAIRES DE TRAVAIL ALTERNANTS CHEZ DES PERSONNELS DE SOINS ET RYTHMICITÉ CIRCADIENNE}

Les personnels du secteur hospitalier (infirmières et aides-soignantes) auxquels nous nous intéressons dans cette étude font partie des $17 \%$ de salariés soumis en France à une organisation du travail en horaires alternants. Des études récentes comparant les conséquences de différents types d'horaires alternants, spécifiquement auprès de ces personnels, décrivent des rythmes circadiens moins robustes et moins amples chez des infirmières travaillant en nuits fixes, comparées à celles exerçant sur d'autres postes (Kang, Miao, Tseng, Sithole, \& Chung, 2015). La somnolence est plus importante sur le poste de nuit par rapport à celle observée sur les postes de jour, et cela indépendamment du type d'horaires alternants et de la durée des postes ( 8 heures ou 12 heures). Elle est associée à un sommeil fragmenté et une dette chronique de sommeil (Costa, Anelli, Castellini, Fustinoni, \& Neri, 2014 ; Garde, Hansen, \& Hansen, 2009 ; Rathore, Shukka, Singh, \& Tiwari, 2012 ; Schaefer, Williams, \& Zee, 2012). Cependant, malgré une somnolence et une fatigue accrues lors du poste de nuit se traduisant par une baisse de la productivité, la vigilance et les capacités de raisonnement des infirmières ne seraient pas affectées (Keller, 2009 ; Reinke, Özbay, Dieperink, \& Tulleken, 2015).

Par ailleurs, plusieurs travaux ont examiné l'impact de l'organisation du travail sur l'évolution de variables physiologiques et psychologiques selon les caractéristiques horaires. Ainsi, chez des infirmières, des rotations en 3 × 8 accentuent la dette et la fragmentation du sommeil (Garde et al., 2009) et ce d'autant plus que les rotations se font dans le sens antihoraire (Costa et al., 2014). Des salariés postés rapportent une baisse de vigilance en fin de matinée si la prise de poste du matin se fait avant 6 heures plutôt qu'après 7 heures, et en début d'après-midi avec des postes de 12 heures plutôt que de 8 heures (Tucker, Barton, \& Folkard, 1996 ; Tucker, Smith, Macdonald, \& Folkard, 1998). Des indicateurs subjectifs et objectifs de stress sont par ailleurs significativement augmentés pour 
des postes démarrant tôt le matin (Bostock \& Steptoe, 2013). De plus, le niveau de vigilance baisse alors que celui de tension subjective augmente avec le nombre d'heures en poste chez des contrôleurs aériens (Mélan, Galy, \& Cariou, 2007). De manière similaire, des infirmières travaillant en postes de jour de 12 heures produisent des évaluations médicales différentes en début et en fin de poste, parallèlement à une baisse de vigilance et une augmentation du stress perçu (McClelland, Switzer, \& Pilcher, 2013). Ces résultats soulignent la modulation des rythmes, notamment de la vigilance, par des paramètres des horaires de travail (direction de la rotation des postes, durée des postes...).

\section{I.3. Horaires DE TRAVAIL ET CHARGE DE TRAVAIL}

D'autres travaux décrivent, chez des opérateurs en $3 \times 8$, des modulations au cours du poste, notamment une tension subjective et une fréquence cardiaque significativement augmentées la première heure de chaque poste comparée aux autres heures du poste, et cela même lors du poste de nuit (Cariou, Galy, \& Mélan, 2008). Ces variations inhabituelles se superposent à une variation circadienne plus globale et refléteraient une charge de travail accrue en début de poste. Cette augmentation de charge de travail serait caractéristique de la prise en charge d'un processus dynamique nécessitant une appréciation de l'état du système sur le moment et une programmation des opérations à effectuer sur le temps du poste. La même interprétation avait été proposée pour rendre compte d'une fréquence de consultation de pages écran significativement supérieure durant la première heure du poste par rapport au reste du poste chez des opérateurs supervisant également un processus dynamique (Andorre \& Quéinnec, 1998).

De manière similaire, chez les personnels de soins, la planification des différentes tâches et les conséquences en termes de charge de travail ne sont pas homogènes sur la journée de 24 heures, et diffèrent entre hôpitaux et milieu (urbain versus rural ; Teasley et al., 2007) et même entre pays. Ainsi, un indicateur indirect de la charge de travail, le niveau d'activité physique au travail, est supérieur chez des infirmières et aidessoignantes polonaises travaillant en horaires alternants que chez celles en horaires fixes de jour (Peplonska, Bukowska, \& Sobala, 2014). De même, chez des infirmières japonaises, la courbe d'activité motrice n'est pas en phase avec la courbe circadienne typique de la fréquence cardiaque (minimum la nuit), alors qu'une telle relation est observée chez des infirmières suisses (Nicoletti et al., 2015). Une étude française de Cazabat, Barthe et Cascino (2008) indique que, sur le poste de nuit, les infirmières tendraient à procéder à des régulations de leur activité, se traduisant par une baisse de l'activité physique et une diminution des demandes de collaborations (celles-ci pouvant s'avérer paradoxalement coûteuses). Ces travaux suggèrent que la charge de travail varie en fonction de plusieurs facteurs caractérisant l'activité de travail, tels que les équipements, la planification des tâches et les procédures, la composition des équipes ou encore les horaires de travail (Barthe, Quéinnec, \& Verdier, 2004 ; Estryn-Behar 
\& Fouillot, 1990 ; Farmer, West, Whyte, \& Maclean, 2005 ; McNeely, 2005 ; Sallinen \& Kecklund, 2010).

Chez les personnels de soins, tout comme pour d'autres populations, les conséquences des horaires de travail alternants et d'une charge de travail élevée sur la performance et, à plus long terme, sur la santé varient également selon des éléments personnels (âge, expérience professionnelle, situation familiale, etc.), comportementaux (habitudes de sommeil, chronotype, etc.), motivationnels et de santé physique et mentale (Boudreau, Dumont, \& Boivin, 2013 ; Costa, 1995 ; Griffiths, Mackey, \& Adamson, 2011 ; Keller, 2009 ; Ota et al., 2005 ; Rathore et al., 2012 ; Reinke et al., 2015). Plus particulièrement, une charge de travail élevée et un sommeil perturbé, typiquement associé au travail en horaires alternants, sont prédictifs d'une fatigue importante, voire de troubles de la santé (Drake, Roehrs, Richardson, Walsh, \& Roth, 2004 ; Folkard, Lombardi, \& Tucker, 2005 ; Tepas \& Carvalhais, 1990).

\section{I.4. OBJECTIFS ET HYPOTHĖSES}

En référence à ces travaux, l'objectif de notre étude consiste à tester l'hypothèse selon laquelle les variations de la charge de travail se révéleront également à travers les perceptions qu'ont les salariés de leur environnement de travail en matière d'exigences (demandes psychologiques et physiques, interférences de la vie hors travail dans le travail) et de ressources mobilisables pour y faire face (contrôle, soutien social, ressources techniques et humaines). Nous faisons alors l'hypothèse que les perceptions de l'environnement de travail résultent de relations complexes entre le poste travaillé et le moment du poste observé. Ainsi, d'un côté, la baisse nocturne de l'état physiologique général, bien documentée chez des salariés postés (Ackerstedt et al., 2004 ; Garde et al., 2009 ; Griffiths et al., 2011) incite à penser que, lors du poste de nuit, le travail serait associé à des perceptions reflétant la présence d'une forte tension professionnelle. D'un autre côté, des études ergonomiques décrivent une organisation du poste de nuit traduisant une activité de travail moins intense centrée sur des aspects de surveillance (Gadbois, 1980 ; Gadbois \& Quéinnec, 1984) ce qui pourrait, au contraire, laisser penser qu'il existe une moindre tension professionnelle comparée à celle perçue au cours des postes de la journée. Cette étude devra permettre de proposer des éléments de réponse sur ce point. Par ailleurs, chez les personnels de soins tout comme pour les opérateurs supervisant un processus dynamique, la tension devrait être plus importante sur la première heure de chacun des postes de travail qu'en milieu ou fin de poste. En début du poste, le personnel doit s'investir dans le recueil d'informations liées à la relève de poste avec l'équipe précédente, notamment en ce qui concerne l'état des différents patients tout en faisant face aux tâches courantes à effectuer et celles à prévoir sur le temps du poste. Finalement, l'effort physique important caractérisant les métiers du soin, accentué encore par une somnolence nocturne croissante au fil des heures du poste de nuit, pourrait se traduire par une augmentation des demandes physiques perçues en cours du poste de nuit. Inversement, la 
composition variable des équipes selon le poste, et plus particulièrement des effectifs plus réduits sur le poste de nuit une fois la relève du poste passée, pourrait se traduire par une évolution inverse du soutien social entre le début et la fin du poste de nuit. Pour tester ces hypothèses, nous avons construit un dispositif de recueil de données permettant une évaluation en temps réel et répétée au cours des différents postes de travail.

\section{MÉTHODOLOGIE DE L’ÉTUDE}

L'étude présentée intègre un programme centré sur les relations entre la charge de travail et la santé chez différentes populations du domaine du soin et du domaine aéronautique. Le protocole complet inclut le dispositif d'évaluation in situ de la présente étude (trois mesures au cours de chacun des postes occupés) ainsi qu'une évaluation plus globale des perceptions de l'environnement de travail et de la santé réalisée en dehors du temps de travail (non détaillée ici).

\section{II.1. CADRE DE L'ÉTUDE}

La présente étude concernant la population des personnels de soins a été réalisée dans un CHU recouvrant 10 établissements, accueillant 3800 médecins et 10600 personnels dont environ 6500 personnels de soins. Organisé autour de 16 pôles médicaux, l'établissement assure 230000 hospitalisations par an (2 880 lits) et 660000 consultations. Après avoir obtenu l'accord de la responsable de la Direction des soins Mission recherche -, nous avons rencontré les responsables des différents pôles retenus pour l'étude et nous avons pu définir avec eux les conditions de réalisation de celle-ci (services retenus, profil des personnels, conditions de dépôt et de retour des questionnaires...). L'étude a été présentée aux personnels soit par le cadre infirmier responsable du service soit par un des membres de l'équipe de recherche. Outre les objectifs de la recherche, les conditions de volontariat et de confidentialité des données ont été rappelées. À cette fin, le retour des questionnaires anonymisés s'est effectué par courrier postal sous enveloppe préaffranchie à l'adresse du laboratoire de recherche.

Nous avons déposé 350 exemplaires du protocole complet. Avec 119 répondants (cadres infirmiers, infirmiers, aides soignants, technicien de laboratoire), le taux de retour $(34 \%)$ se situe au-delà du pourcentage habituel de retours à un questionnaire en l'absence de relance (20 à $30 \%$ ). Ont été retenus ici les participants remplissant l'ensemble des conditions requises : exercer habituellement en horaires alternants, avoir renseigné le questionnaire lors des trois phases d'observation définies pour un même poste (début, milieu et fin de poste). Douze participants ont été écartés des analyses : neuf en raison d'un cycle d'alternance en $2 \times 12$ et trois en raison d'un nombre élevé de réponses manquantes. Au bilan, nous disposons de 86 observations issues de 38 participants. 


\section{II.2. PARTicipants}

L'échantillon retenu pour la présente étude est constitué de 38 personnels hospitaliers, 24 infirmières (IDE) et 14 aides-soignantes (AS) affectées dans cinq services (digestif, locomoteur, ophtalmologie, cardiologie dont chirurgie et soins intensifs cardiologie et maxillo-faciale). Dans ces services, les personnels travaillent pour la plupart d'entre eux habituellement en $2 \times 8$ ou en $3 \times 8$, certains sont en horaires fixes de jour ou en horaires fixes de nuit. Au vu des objectifs de l'étude, n'ont été retenus que les personnels travaillant habituellement en $2 \times 8(\mathrm{~N}=19$ avec 8 AS et $11 \mathrm{IDE})$ et en $3 \times 8$ $(\mathrm{N}=19 \text { avec } 6 \text { AS et } 13 \mathrm{IDE})^{1}$. La représentation des différents services est sensiblement identique pour les observations réalisées sur les différents postes. L'âge moyen est de 35.8 ans (e.t. $=10.73$ ) et varie de 22 à 57 ans.

\section{II.3. MATÉRIEL}

Les perceptions de l'environnement de travail sont habituellement appréhendées à l'aide du "fob Content Questionnaire" (JCQ, Karasek et al., 1998). Ce questionnaire intègre trois des cinq dimensions considérées dans notre étude (demandes, contrôle, soutien social). La consigne générale et la formulation des items qui composent ce questionnaire réfèrent à une période de temps non définie, pouvant s'étaler de quelques semaines à plusieurs mois suscitant ainsi une évaluation globale ou "moyenne ». Le participant peut alors formuler ses réponses à partir des conditions de travail telles qu'il les a perçues le jour même, la veille, sur une semaine ou globalement sur les six derniers mois (Johnston, Beedie, \& Jones, 2006). L'utilisation d'une telle mesure générale ne permet pas de répondre à notre objectif d'évaluation de la variabilité des perceptions à plus court terme, en fonction du poste travaillé et/ou du moment du poste observé. Certains outils permettent une mesure in situ de la charge mentale ou charge cognitive, en particulier le NASA-TLX (Hart \& Staveland, 1988). Cependant, les six items composant cette échelle, évaluant respectivement les demandes (mentale, physique ou temporelle), la performance perçue, l'effort et le niveau de frustration, ne recouvrent pas les dimensions que nous souhaitions évaluer.

Nous avons développé dans le cadre de notre programme de recherche un questionnaire spécifique (comportant 56 items) adapté à nos objectifs (cf. annexe I). Pour évaluer les demandes auxquelles les personnels doivent répondre, trois échelles sont prises en compte :

- les demandes psychologiques (19 items ; $\alpha=.96)$;

- les demandes physiques ( 6 items ; $\alpha=.88$ );

- les interférences de la vie hors travail sur le travail (5 items; $\alpha=.94)^{2}$.

1. L'indépendance des facteurs " horaires " et "statut " $\left(X^{2}=0.186, p<.666\right)$ ainsi que celle des facteurs "statut " et "poste" $\left(X^{2}=.889, p<.641\right)$ sont confirmées.

2. Les réponses obtenues auprès de cet échantillon sur les items relatifs aux interférences entre le travail et la vie hors travail étant non discriminantes (la quasi-totalité des réponses se situant sur une seule modalité), cette dimension n'a pas été retenue pour les analyses. 
Les ressources perçues relèvent de trois catégories :

- les ressources de contrôle avec l'autonomie ( 4 items ; $\alpha=.85$ ) et l'utilisation et le développement des compétences ( 8 items ; $\alpha=.89$ );

- le soutien social avec le soutien des supérieurs ( 3 items ; $\alpha=.87$ ) et le soutien des collègues ( 4 items ; $\alpha=.82$ );

- les ressources techniques et humaines ( 5 items ; $\alpha=.90$ ).

La consigne écrite (cf. annexe II) invite les participants à référer leurs réponses au contexte temporel de l'heure venant de s'écouler (début de poste : "Vous êtes à votre poste de travail depuis maintenant environ 1 heure"; milieu de poste : "Vous êtes maintenant à peu près à la moitié de votre journée de travail"; fin du poste : "D'ici environ 1 heure vous aurez terminé votre journée de travail»). Pour l'ensemble des items, les réponses sont portées sur une échelle en 6 points de 1 "pas du tout présent " à 6 "très souvent présent ". Les différents scores sont construits par calcul de la moyenne des réponses aux items de la dimension et varient donc de 1 à 6. Plus la moyenne est élevée, plus les niveaux de demandes, d'interférences et de ressources perçues sont importants. La fiabilité interne des sept échelles a été évaluée par calcul des alphas de Cronbach : les valeurs obtenues s'échelonnant de .82 à .96 permettent de conclure à la fiabilité des mesures.

\section{II.4. ProcÉdure}

Fourni en trois exemplaires pour chaque poste de travail, le questionnaire devait être complété trois fois au cours d'un même poste de travail : respectivement une heure après le début du poste, en milieu de poste et une heure avant la fin du poste (cf. consigne en annexe II). Les personnels travaillant en 2x8 devaient remplir deux jeux de trois questionnaires (un pour le poste du matin et un pour le poste de l'après-midi), les personnels en $3 \times 8$ trois jeux de questionnaires (pour les postes du matin, de l'aprèsmidi et de nuit). Vingt-neuf répondants ont fourni un jeu complet de questionnaires et 9 n'ont répondu que pour une partie des postes (le poste non renseigné se distribuant aléatoirement). N'ont été retenues que les observations complètes sur un même poste c'est-à-dire les questionnaires renseignés trois fois au cours du poste. L'indépendance des réponses au facteur " horaires » $(2 \times 8$ versus $3 \times 8)$ ayant été testée par une ANOVA, nous avons pris en considération, pour le poste du matin et de l'après-midi, les questionnaires renseignés tant par les personnels en $2 \times 8$ qu'en $3 \times 8$ (32 questionnaires pour le poste du matin et 35 pour le poste de l'aprèsmidi) et, pour le poste de nuit, les 19 observations produites par 13 des 19 personnels en $3 \times 8$ et par 6 personnels occasionnellement mobilisés sur un poste de nuit.

\section{II.5. Plan D’ANAlyse DES DONNÉES}

Afin de tester les effets principaux ainsi qu'une possible interaction "postes x phases", nous avons réalisé des ANOVAs à deux facteurs 
répétés : le poste travaillé et la phase du poste (début (D), milieu (M) et fin (F) de poste). Une première ANOVA a été réalisée sur les observations obtenues sur les trois postes (matin $(\mathrm{Ma})$, après-midi $(\mathrm{Am})$ et nuit $(\mathrm{Nu})$; ANOVA 3x3; $=20$ ). Dans une seconde ANOVA, a été inclus l'ensemble des observations recueillies sur les postes du matin $(\mathrm{Ma})$ et de l'après-midi (Am) (ANOVA $2 \times 3 ; n=38$ ). Les analyses post-hoc ont été effectuées avec le test de Bonferroni.

\section{RÉSULTATS}

\section{III.1. EXAMEN DES DIFFÉRENCES ENTRE LES PHASES}

L'hypothèse d'une évolution des perceptions au cours du poste (cf. tableau 1 ci-après) est confirmée pour les demandes physiques. Ainsi, chez les personnels ayant renseigné le questionnaire pour chacun des trois postes, la variation des demandes physiques $(F[2,30]=6.478 ; p<.01$, $\left.\eta^{2}=.302\right)$ se traduit par des niveaux plus faibles en début de poste $(M=3.05)$ qu'en milieu $(M=3.39, p<.04)$ et en fin de poste $(M=3.48, p<.04)$. Cette hypothèse n'est pas vérifiée pour les demandes psychologiques et les différentes ressources prises en considération dans l'étude (tous les $\mathrm{F}$ étant non significatifs).

TABLEAU 1 : Effets principaux du facteur "phase ", ANOVAs sur les trois postes $(\mathrm{F}$, effectif $=N$, moyenne $=$ Moy et erreur-standard $=\mathrm{es})$.

TABLE 1: Main effects of the factor "phase", ANOVAs including the three shitfs $(F$, number of participants $=\mathrm{N}$, mean $=$ Moy and standard error $=e s)$.

\begin{tabular}{|l|l|c|c|c|c|c|c|c|}
\hline & \multicolumn{1}{|c|}{ ANOVA 3x3 } & & \multicolumn{2}{c|}{ Début } & \multicolumn{2}{c|}{ Milieu } & \multicolumn{2}{c|}{ Fin } \\
\hline & & $\mathbf{N}$ & Moy & es & Moy & es & Moy & es \\
\hline $\begin{array}{l}\text { Demandes } \\
\text { physiques }\end{array}$ & $\begin{array}{l}F[2,30]=6.478 ; \\
p<.01, \eta^{2}=.302\end{array}$ & 16 & 3.05 & .16 & 3.39 & .21 & 3.48 & .23 \\
\hline $\begin{array}{l}\text { Demandes } \\
\text { psycho. }\end{array}$ & $\begin{array}{l}F[2,32]=1.495 ; \\
p<.24, n s\end{array}$ & 17 & 3.44 & .11 & 3.33 & .16 & 3.24 & .17 \\
\hline $\begin{array}{l}\text { Utilisation } \\
\text { compétences }\end{array}$ & $\begin{array}{l}F[2,30]=0.691 ; \\
p<.50, n s\end{array}$ & 16 & 3.97 & .15 & 3.86 & .17 & 3.91 & .18 \\
\hline Autonomie & $F[2,32]=0.175 ; p<.75, n s$ & 17 & 4.07 & .13 & 3.99 & .14 & 4.00 & .17 \\
\hline $\begin{array}{l}\text { Ress. Tech. } \\
\text { et humaines }\end{array}$ & $\begin{array}{l}F[2,30]=1.354 ; \\
p<.27, n s\end{array}$ & 16 & 4.10 & .20 & 3.95 & .20 & 3.90 & .23 \\
\hline $\begin{array}{l}\text { Soutien } \\
\text { des collègues }\end{array}$ & $\begin{array}{l}F[2,30]=0.127 ; \\
p<.84, n s\end{array}$ & 16 & 3.63 & .17 & 3.64 & .24 & 3.60 & .21 \\
\hline $\begin{array}{l}\text { Soutien } \\
\text { des supérieurs }\end{array}$ & $\begin{array}{l}F[2,32]=0.814 ; \\
p<.43, n s\end{array}$ & 17 & 4.30 & .19 & 4.30 & .16 & 4.38 & .18 \\
\hline
\end{tabular}


On obtient un résultat identique lorsque l'analyse concerne les réponses obtenues lors des seuls postes du matin et de l'après-midi (cf. tableau 2 cidessous). En effet, les résultats confirment l'augmentation des demandes physiques $\left(F[2,56]=4.183 ; p<.02, \eta^{2}=.130\right)$ passée la $1^{\text {re }}$ heure du poste (respectivement $M=3.05, M=3.70$, et $M=3.56$; post-hoc Début/Milieu $p<.001 ;$ Début/Fin $p<.03)$.

TABlEau 2 : Effets principaux du facteur "phase ", ANOVAs sur les postes du matin et de l'après-midi $(\mathrm{F}$, effectif $=N$, moyenne $=$ Moy et erreur-standard $=\mathrm{es}$ ).

TABLE 2: Main effects of the factor "phase", ANOVAs including the morningand afternoon-shifts $(F$, number of participants $=\mathrm{N}$, mean $=$ Moy and standard error $=e s)$.

\begin{tabular}{|l|l|c|c|c|c|c|c|c|}
\hline & \multicolumn{1}{|c|}{ ANOVA 2x3 } & & \multicolumn{2}{c|}{ Début } & \multicolumn{2}{c|}{ Milieu } & \multicolumn{2}{c|}{ Fin } \\
\hline & & $\mathbf{N}$ & Moy & es & Moy & es & Moy & es \\
\hline $\begin{array}{l}\text { Demandes } \\
\text { physiques }\end{array}$ & $\begin{array}{l}F[2,54]=9.884 ; \\
p<.000, \eta^{2}=.014\end{array}$ & 28 & 3.05 & .15 & 3.70 & .16 & 3.56 & .19 \\
\hline $\begin{array}{l}\text { Demandes } \\
\text { psycho. }\end{array}$ & $\begin{array}{l}F[2,56]=4.183 ; \\
p<.02, \eta^{2}=.130\end{array}$ & 29 & 3.48 & .15 & 3.68 & .13 & 3.33 & .16 \\
\hline $\begin{array}{l}\text { Utilisation } \\
\text { compétences }\end{array}$ & $\begin{array}{l}F[2,54]=3.009 ; \\
p<.06, n s\end{array}$ & 28 & 3.78 & .15 & 4.01 & .12 & 3.77 & .16 \\
\hline Autonomie & $F[2,56]=0.026 ; p<.92, n s$ & 29 & 3.83 & .11 & 3.81 & .11 & 3.83 & .14 \\
\hline $\begin{array}{l}\text { Ress. Tech. } \\
\text { et humaines }\end{array}$ & $\begin{array}{l}F[2,54]=0.220 ; \\
p<.78, n s\end{array}$ & 28 & 4.10 & .16 & 4.03 & .13 & 4.06 & .14 \\
\hline $\begin{array}{l}\text { Soutien } \\
\text { des collègues }\end{array}$ & $\begin{array}{l}F[2,52]=2.151 ; \\
p<.13, n s\end{array}$ & 27 & 3.53 & .17 & 3.73 & .17 & 3.59 & .15 \\
\hline $\begin{array}{l}\text { Soutien } \\
\text { des supérieurs }\end{array}$ & $\begin{array}{l}F[2,56]=0.642 ; \\
p<.50, n s\end{array}$ & 29 & 4.20 & .14 & 4.28 & .12 & 4.30 & .12 \\
\hline
\end{tabular}

Cette seconde analyse fait également apparaître, en plus des variations significatives des demandes physiques rapportées ci-dessus, des différences relatives aux demandes psychologiques $\left(F[2,56]=4.183 ; p<.02, \eta^{2}=.130\right)$. Ces dernières sont plus faibles en fin de poste qu'en début et milieu de poste (respectivement $M=3.33, M=3.48$ et $M=3.68$ ), avec une différence significative entre le milieu et la fin du poste $(p<.02)$. Aucune variation au cours du poste n'est observée concernant les différentes dimensions de ressources (tous les $\mathrm{F}$ étant non significatifs).

\section{III.2. EXAMEN DES DIFFÉRENCES ENTRE LES POSTES}

L'examen des effets principaux du poste permet de confirmer l'hypothèse d'un effet du poste sur les perceptions de l'environnement de travail.

Dans le tableau 3 ci-dessous, on note tout d'abord une différence significative en ce qui concerne les demandes physiques $(F[2,30]=8.56$; 
$\left.p<.003, \eta^{2}=.363\right)$, qui sont plus faibles lors du poste de nuit $(M=2.93)$ que lors du poste du matin $(M=3.43, p<.06)$ et du poste d'après-midi $(M=3.57, p<.005)$. Une tendance similaire $(F[2,32]=3.142 ; p<.08$, $\left.\eta^{2}=.164\right)$ s'observe pour les demandes psychologiques (respectivement, $M(N u)=3.03, M(M a t)=3.48, M(A m)=3.50 ;$ post - hoc $M a t / N u p<.08)$. Les résultats indiquent également un effet significatif du poste sur la dimension " autonomie » $\left(F[2,32]=4.738 ; p<.02, \eta^{2}=.228\right)$ : la nuit, les personnels perçoivent en moyenne davantage d'autonomie $(M=4.34)$ en comparaison des postes du matin $(M=3.78)$ et de l'après-midi $(M=3.94)$, mais l'ensemble des tests post-hoc n'est pas significatif. Inversement, la nuit, le soutien social des collègues $\left(F[2,30]=4.702 ; p<.02, \eta^{2}=.239\right)$ est perçu comme étant plus faible $(M=3.19)$ que le matin $(M=3.98)$ et l'après-midi $(M=3.69)$, même si les tests post-hoc (Mat/Nu et $\mathrm{Amidi} / \mathrm{Nu}$ ) s'avèrent non significatifs. Les perceptions d'utilisation des compétences $(F[2,30]=0.310 ; p<.72, n s)$, de soutien des supérieurs $(F[2,32]=0.986$; $p<.38$, ns $)$ et des ressources techniques et humaines $(F[2,30]=0.346$; $p<.71, n s)$ sont similaires quel que soit le poste.

TABLEAU 3 : Effets principaux du facteur "poste ", ANOVAs sur les trois postes $(\mathrm{F}$, effectif $=N$, moyenne $=$ Moy et erreur-standard $=\mathrm{es})$.

TABLE 3: Main effects of the factor "shift", ANOVAs including the three shifts $(F$, number of participants $=\mathrm{N}$, mean $=M o y$ and standard error $=e s)$.

\begin{tabular}{|l|l|c|c|c|c|c|c|c|}
\hline & \multicolumn{1}{|c|}{ ANOVA 3x3 } & & \multicolumn{2}{c|}{ Matin } & \multicolumn{2}{c|}{ A-midi } & \multicolumn{2}{c|}{ Nuit } \\
\hline & & $\mathbf{N}$ & Moy & es & Moy & es & Moy & es \\
\hline $\begin{array}{l}\text { Demandes } \\
\text { physiques }\end{array}$ & $\begin{array}{l}F[2,30]=8.56 ; \\
p<.003, \eta^{2}=.363\end{array}$ & 16 & 3.43 & .20 & 3.57 & .21 & 2.93 & .22 \\
\hline Demandes psycho. & $\begin{array}{l}F[2,32]=3.142 ; \\
p<.08, \eta^{2}=.164\end{array}$ & 17 & 3.48 & .15 & 3.50 & .19 & 3.03 & .20 \\
\hline $\begin{array}{l}\text { Utilisation } \\
\text { compétences }\end{array}$ & $\begin{array}{l}F[2,30]=0.310 ; \\
p<.72, n s\end{array}$ & 16 & 3.83 & .22 & 3.94 & .16 & 3.96 & .18 \\
\hline Autonomie & $\begin{array}{l}F[2,32]=4.738 ; \\
p<.02, \eta^{2}=.228\end{array}$ & 17 & 3.78 & .16 & 3.94 & .14 & 4.34 & .19 \\
\hline $\begin{array}{l}\text { Ress. Tech. } \\
\text { et humaines }\end{array}$ & $\begin{array}{l}F[2,30]=0.346 ; \\
p<.71, n s\end{array}$ & 16 & 4.11 & .21 & 3.92 & .26 & 3.93 & .27 \\
\hline $\begin{array}{l}\text { Soutien } \\
\text { des collègues }\end{array}$ & $\begin{array}{l}F[2,30]=4.702 ; \\
p<.02, \eta^{2}=.239\end{array}$ & 16 & 3.98 & .17 & 3.69 & .25 & 3.19 & .32 \\
\hline $\begin{array}{l}\text { Soutien } \\
\text { des supérieurs }\end{array}$ & $\begin{array}{l}F[2,32]=0.986 ; \\
p<.38, n s\end{array}$ & 17 & 4.19 & .19 & 4.38 & .19 & 4.41 & 322 \\
\hline
\end{tabular}

Si cette première analyse souligne la spécificité du poste de nuit (moins de demandes physiques et psychologiques, plus d'autonomie et un moindre soutien social) comparé aux postes tenus sur la journée (matin et après-midi), l'analyse portant sur ces derniers (cf. tableau 4 ci-après) doit permettre d'affiner les différences entre les deux postes se déroulant sur la journée. 
TABLEAU 4 : Effets principaux du facteur "poste ", ANOVAs sur les postes du matin et de l'après-midi ( $\mathrm{F}$, effectif $=N$, moyenne $=$ Moy et erreur standard $=\mathrm{es})$.

TABLE 4: Main effects of the factor "shift", ANOVAs including the morningand afternoon-shifts $(F$, number of participants $=\mathrm{N}$, mean $=$ Moy and standard error=es).

\begin{tabular}{|l|l|c|c|c|c|c|}
\hline & \multicolumn{1}{|c|}{ ANOVA 2x3 } & & \multicolumn{2}{c|}{ Matin } & \multicolumn{2}{c|}{ A-midi } \\
\hline & & $N$ & Moy & es & Moy & es \\
\hline $\begin{array}{l}\text { Demandes } \\
\text { physiques }\end{array}$ & $\begin{array}{l}F[1,27]=0.382 ; \\
p<.54, n s\end{array}$ & 28 & 3.40 & .15 & 3.48 & .17 \\
\hline $\begin{array}{l}\text { Demandes } \\
\text { psycho. }\end{array}$ & $\begin{array}{l}F[1.28]=0.668 ; \\
p<.42, n s\end{array}$ & 29 & 3.44 & .13 & 3.55 & .17 \\
\hline $\begin{array}{l}\text { Utilisation } \\
\text { compétences }\end{array}$ & $\begin{array}{l}F[1,27]=1.028 ; \\
p<.32, n s\end{array}$ & 28 & 3.78 & .16 & 3.93 & .14 \\
\hline $\begin{array}{l}\text { Autonomie } \\
\text { Rens }\end{array}$ & $\begin{array}{l}F[1.28]=7.024 ; \\
p<.01, \eta^{2}=.201\end{array}$ & 29 & 3.69 & .11 & 3.96 & .10 \\
\hline $\begin{array}{l}\text { Ress. Tech. } \\
\text { et humaines }\end{array}$ & $\begin{array}{l}F[1,27]=0.269 ; \\
p<.61, n s\end{array}$ & 28 & 4.01 & .15 & 4.11 & .17 \\
\hline $\begin{array}{l}\text { Soutien } \\
\text { des collègues }\end{array}$ & $\begin{array}{l}F[1,26]=0.471 ; \\
p<.50, n s\end{array}$ & 27 & 3.66 & .16 & 3.56 & .18 \\
\hline $\begin{array}{l}\text { Soutien } \\
\text { des supérieurs }\end{array}$ & $\begin{array}{l}F[1,28]=3.618 ; \\
p<.07, n s\end{array}$ & 29 & 4.15 & .13 & 4.37 & .13 \\
\hline
\end{tabular}

Ces deux postes se différencient significativement uniquement en matière d'autonomie $\left(F[1.28]=7.024 ; p<.01, \eta^{2}=.201\right)$ plus élevée lors du poste de l'après-midi $(M=3.96)$ que lors du poste du matin $(M=3.69)$. Par contre, aucune différence significative n'apparaît pour les autres types de ressources ou pour les demandes physiques et psychologiques (tous les $F$ étant non significatifs).

\section{III.3. EXAMEN DE L'Évolution AU COURS DU POSTE}

Dans ce paragraphe, nous présentons d'abord les effets d'interaction pour les ANOVAs 3x3, puis 2x3. Ensuite, nous décomposerons ces effets pour le poste de nuit à partir de l'ANOVA portant sur les trois postes. Les effets d'interaction pour les postes du matin et de l'après-midi étant largement concordants dans les deux analyses, ils seront présentés à partir de l'ANOVA $2 \times 3$. La Figure 1 illustre les différences d'évolution des perceptions au cours du poste et selon les postes (seules les dimensions donnant lieu à des variations significatives sont visualisées).

L'hypothèse d'une évolution différenciée en cours de poste selon le poste travaillé (matin, après-midi, nuit) se confirme (cf. tableau 5 ciaprès, ANOVA $3 \times 3$ ) en ce qui concerne le soutien social des collègues $\left(F[4,60]=4.806 ; p<.01, \eta^{2}=.243\right)$. Une tendance s'observe également concernant les différences de demandes physiques $(F[4,60]=3.067$; 
$\left.p<.06, \eta^{2}=.170\right)$ et de demandes psychologiques $(F[4,64]=2.901 ; p<.06$, $\left.\eta^{2}=.153\right)$. Les dimensions du contrôle et les ressources techniques et humaines ne donnent pas lieu à une interaction significative.

TABlEAu 5 : Interaction "poste " $x$ "phase ", ANOVAs sur les trois postes $(\mathrm{F}$, effectif $=N$, moyenne $=$ Moy et erreur-standard $=\mathrm{es})$.

TABLE 5: Combined effects of "shift" and "phase", ANOVAs including the three shifts $(F$, number of participants $=\mathrm{N}$, mean $=$ Moy and standard error $=e s)$.

\begin{tabular}{|c|c|c|c|c|c|c|c|c|c|}
\hline & \multirow[t]{2}{*}{ ANOVA $3 \times 3$} & & & \multicolumn{2}{|c|}{$\mathbf{D}$} & \multicolumn{2}{|c|}{$\mathbf{M}$} & \multicolumn{2}{|c|}{$\mathbf{F}$} \\
\hline & & & $\mathbf{N}$ & Moy & e.s. & Moy & e.s. & Moy & e.s. \\
\hline \multirow{3}{*}{$\begin{array}{l}\text { Demandes } \\
\text { physiques }\end{array}$} & \multirow{3}{*}{$\begin{array}{l}F[4,60]=3.067 \\
p<.06, \eta^{2}=.170\end{array}$} & Mat & 16 & 2,87 & .23 & 3,84 & .25 & 3,57 & .31 \\
\hline & & $\mathrm{AM}$ & 16 & 3,45 & .17 & 3,61 & .26 & 3,66 & .29 \\
\hline & & $\mathrm{Nu}$ & 16 & 2,84 & .26 & 2,73 & .28 & 3,22 & .20 \\
\hline \multirow{3}{*}{$\begin{array}{l}\text { Demandes } \\
\text { psycho. }\end{array}$} & \multirow{3}{*}{$\begin{array}{l}F[4,64]=2.901 ; \\
p<.06, \eta^{2}=.153\end{array}$} & Mat & 17 & 3,47 & .20 & 3,74 & .18 & 3,24 & .20 \\
\hline & & $\mathrm{AM}$ & 17 & 3,68 & .19 & 3,49 & .21 & 3,33 & .24 \\
\hline & & $\mathrm{Nu}$ & 17 & 3,16 & .17 & 2,77 & .21 & 3,17 & .31 \\
\hline \multirow{3}{*}{$\begin{array}{l}\text { Utilisation } \\
\text { compétences }\end{array}$} & \multirow{3}{*}{$\begin{array}{l}F[4,60]=0.145 ; \\
p<.90, \eta^{2}=.009\end{array}$} & Mat & 16 & 3,81 & .25 & 3,92 & .24 & 3,78 & .25 \\
\hline & & $\mathrm{AM}$ & 16 & 3,97 & .20 & 3,86 & .17 & 3,99 & .18 \\
\hline & & $\mathrm{Nu}$ & 16 & 4,13 & .19 & 3,80 & .20 & 3,96 & .21 \\
\hline \multirow[t]{3}{*}{ Autonomie } & \multirow{3}{*}{$\begin{array}{l}F[4,64]=1.070 ; \\
p<.37, \eta^{2}=.067\end{array}$} & Mat & 17 & 3,82 & .20 & 3,73 & .15 & 3,78 & .16 \\
\hline & & $\mathrm{AM}$ & 17 & 3,94 & .13 & 3,97 & .17 & 3,92 & .28 \\
\hline & & $\mathrm{Nu}$ & 17 & 4,44 & .16 & 4,28 & .22 & 4,32 & .26 \\
\hline \multirow{3}{*}{$\begin{array}{l}\text { Ress. Tech. } \\
\text { et humaines }\end{array}$} & \multirow{3}{*}{$\begin{array}{l}F[4,60]=1.369 ; \\
p<.26, \eta^{2}=.084\end{array}$} & Mat & 16 & 4,09 & .26 & 4,04 & .23 & 4,20 & .24 \\
\hline & & $\mathrm{AM}$ & 16 & 4,13 & .29 & 3,87 & .27 & 3,76 & .27 \\
\hline & & $\mathrm{Nu}$ & 16 & 4,09 & .23 & 3,95 & .28 & 3,75 & .35 \\
\hline \multirow{3}{*}{$\begin{array}{l}\text { Soutien } \\
\text { des collègues }\end{array}$} & \multirow{3}{*}{$\begin{array}{l}F[4,60]=4.806 ; \\
p<.01, \eta^{2}=.243\end{array}$} & Mat & 16 & 3,65 & .17 & 4,13 & .21 & 4,17 & .20 \\
\hline & & $\mathrm{AM}$ & 16 & 3,98 & .23 & 3,73 & .30 & 3,38 & .30 \\
\hline & & $\mathrm{Nu}$ & 16 & 3,27 & .29 & 3,06 & .38 & 3,25 & .31 \\
\hline \multirow{3}{*}{$\begin{array}{l}\text { Soutien } \\
\text { des supérieurs }\end{array}$} & \multirow{3}{*}{$\begin{array}{l}F[4,64]=1.369 ; \\
p<.27, \eta^{2}=.079\end{array}$} & Mat & 17 & 4,12 & .20 & 4,09 & .18 & 4,34 & .21 \\
\hline & & $\mathrm{AM}$ & 17 & 4,41 & .25 & 4,42 & .18 & 4,32 & .19 \\
\hline & & $\mathrm{Nu}$ & 17 & 4,37 & .22 & 4,39 & .20 & 4,46 & .26 \\
\hline
\end{tabular}

Les résultats de l'ANOVA réalisée sur les observations du matin et de l'après-midi (ANOVA $2 \times 3$ ) confirment les effets d'interaction du poste et de la phase (cf. tableau 6 ci-après) observés pour le soutien social des collègues $\left(F[2,52]=10.215 ; p<.001, \eta^{2}=.282\right)$ et valident ceux relatifs aux demandes physiques $\left(F[2,54]=4.930 ; p<.01, \eta^{2}=.154\right)$ et aux demandes psychologiques $\left(F[2,56]=3.586 ; p<.05, \eta^{2}=.114\right)$. Il se dégage également un effet d'interaction pour les ressources techniques et humaines 
$\left(F[2,54]=6.351 ; p<.01, \eta^{2}=.190\right)$. Seule la dimension contrôle ne donne lieu à aucune interaction.

TABleau 6 : Interaction "poste " $x$ "phase ", ANOVAs sur les postes du matin et de l'après-midi ( $\mathrm{F}$, effectif $=N$, moyenne $=$ Moy et erreur-standard $=\mathrm{es})$.

TABLE 6: Combined effects of "shift" and "phase", ANOVAs including the morning- and afternoon-shifts $(F$, number of participants $=\mathrm{N}$, mean=Moy and standard error $=e s$ ).

\begin{tabular}{|c|c|c|c|c|c|c|c|c|c|}
\hline & \multirow[t]{2}{*}{ ANOVA $2 \times 3$} & \multirow[b]{2}{*}{$\mathbf{N}$} & & \multicolumn{2}{|c|}{$\mathbf{D}$} & \multicolumn{2}{|c|}{$\mathbf{M}$} & \multicolumn{2}{|c|}{$\mathbf{F}$} \\
\hline & & & & Moy & e.s. & Moy & e.s. & Moy & e.s. \\
\hline \multirow{2}{*}{$\begin{array}{l}\text { Demandes } \\
\text { physiques }\end{array}$} & \multirow{2}{*}{$\begin{array}{l}F[2,54]=4.930 \\
p<.01, \eta^{2}=.154\end{array}$} & 28 & Mat & 2,86 & .18 & 3,85 & . 17 & 3,48 & .21 \\
\hline & & 28 & $\mathrm{AM}$ & 3,24 & .15 & 3,55 & .20 & 3,64 & .22 \\
\hline \multirow{2}{*}{$\begin{array}{l}\text { Demandes } \\
\text { psycho. }\end{array}$} & \multirow{2}{*}{$\begin{array}{l}F[2,56]=3.586 \\
p<.05, \eta^{2}=.114\end{array}$} & 29 & Mat & 3,35 & .17 & 3,77 & .13 & 3,20 & .17 \\
\hline & & 29 & $\mathrm{AM}$ & 3,60 & .16 & 3,59 & .17 & 3,46 & .22 \\
\hline \multirow{2}{*}{$\begin{array}{l}\text { Utilisation } \\
\text { compétences }\end{array}$} & \multirow{2}{*}{$\begin{array}{l}F[2,54]=1.063 \\
p<.35, \eta^{2}=.038\end{array}$} & 28 & Mat & 3,65 & .20 & 4,01 & .15 & 3,68 & .19 \\
\hline & & 28 & $\mathrm{AM}$ & 3,91 & .17 & 4,02 & .15 & 3,86 & .15 \\
\hline \multirow[t]{2}{*}{ Autonomie } & \multirow{2}{*}{$\begin{array}{l}F[2,56]=0.780 ; \\
p<.45, \eta^{2}=.027\end{array}$} & 29 & Mat & 3,76 & .14 & 3,67 & .10 & 3,63 & .14 \\
\hline & & 29 & $\mathrm{AM}$ & 3,91 & .10 & 3,94 & .14 & 4,03 & .20 \\
\hline \multirow{2}{*}{$\begin{array}{l}\text { Ress. Tech. } \\
\text { et humaines }\end{array}$} & \multirow{2}{*}{$\begin{array}{l}F[2,54]=6.351 \\
p<.01, \eta^{2}=.190\end{array}$} & 28 & Mat & 3,86 & .19 & 3,99 & .16 & 4,19 & .17 \\
\hline & & 28 & $\mathrm{AM}$ & 4,33 & .22 & 4,06 & .17 & 3,92 & .17 \\
\hline \multirow{2}{*}{$\begin{array}{l}\text { Soutien } \\
\text { des collègues }\end{array}$} & \multirow{2}{*}{$\begin{array}{l}F[2,52]=10.215 ; \\
p<.001, \eta^{2}=.282\end{array}$} & 27 & Mat & 3,33 & .18 & 3,82 & .19 & 3,83 & .17 \\
\hline & & 27 & $\mathrm{AM}$ & 3,73 & .21 & 3,63 & .20 & 3,31 & .20 \\
\hline \multirow{2}{*}{$\begin{array}{l}\text { Soutien } \\
\text { des supérieurs }\end{array}$} & \multirow{2}{*}{$\begin{array}{l}F[2,56]=0.703 \\
p<.47, \eta^{2}=.024\end{array}$} & 29 & Mat & 4,07 & .14 & 4,13 & .13 & 4,26 & .15 \\
\hline & & 29 & $\mathrm{AM}$ & 4,33 & .17 & 4,42 & .14 & 4,34 & .17 \\
\hline
\end{tabular}

$\mathrm{Au}$ cours du poste de nuit, les demandes physiques augmentent tout au long du poste (respectivement $M=2.84, M=2.73$ et $M=3.22$; post-hoc Début/Fin $p<.07$; Milieu/Fin $p<.01)$ alors que les demandes psychologiques diminuent après la $1^{\text {re }}$ heure de poste $(M(D)=3.16$ et $M(M)=2.77$; post-hoc Début/Milieu $p<.02$ ) avant d'augmenter à nouveau en fin de poste $(M(F)=3.17$, post-hoc Milieu/Fin $p<.10)$. On observe par contre une relative stabilité des perceptions de soutien social des collègues et des ressources techniques et humaines.

Lors du poste du matin, les demandes physiques augmentent passée la $1^{\text {re }}$ heure de poste (respectivement $M=2.86$ et $M=3.85$; tests post-hoc Début/Milieu $p<.001$ ) pour diminuer et atteindre leur niveau le plus bas en fin de poste $(M=3.48$; post-hoc Milieu/Fin $p<.04$; Début/Fin $p<.02)$. On note également une augmentation des demandes psychologiques entre le début $(M=3.35)$ et le milieu du poste $(M=3.77$; post-hoc Début/Milieu $p<.01)$ puis une diminution jusqu'à la fin du poste $(M=3.20$; post-hoc Milieu/Fin $p<.001)$. Le soutien social des collègues augmente quant à lui 
après la $1^{\text {re }}$ heure du poste (respectivement $M=3.33, M=3.82$ et $M=3.83$, tests post-hoc Début/Milieu $p<.001$ et Début/Fin $p<.003$ ). Les ressources techniques et humaines sont quant à elles plus faibles en début $(M=3.86)$ qu'en fin de poste $(M=4.19$, post-hoc Début/Fin $p<.03)$.

Lors du poste de l'après-midi, les demandes physiques augmentent de façon continue au cours du poste (respectivement $M=3.24, M=3.55$ et $M=3.64$; post-hoc Début/Milieu $p<.09$; Début/Fin $p<.05$ ) alors que les demandes psychologiques restent relativement stables. Parallèlement, le soutien social des collègues diminue tout au long du poste (respectivement $M=3.73, M=3.63$ et $M=3.31$; post-hoc Début/Fin $p<.02$ et $M i l i e u / F i n$ $p<.02)$ comme les perceptions liées aux ressources techniques et humaines, elles aussi diminuant tout au long du poste (respectivement $M=4.33$, $M=4.06$ et $M=3.92$; post-hoc Début/Milieu $p<.08$; Début/Fin $p<.03)$.

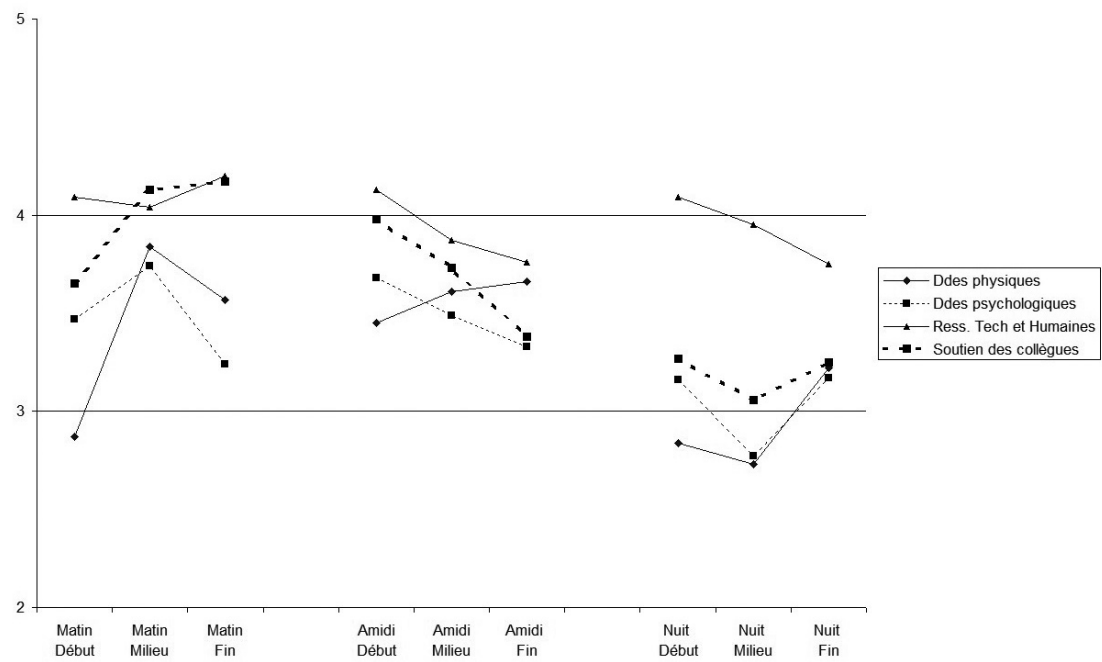

Figure 1 : Évolution des dimensions au cours du poste pour chacun des postes observés (échelle de 1 à 6).

Figure 1:Variations of the different dimensions across the three shift-phases on each shift (scale from 1 to 6 ).

\section{DISCUSSION}

Les résultats obtenus dans cette étude, pris dans leur ensemble, confirment l'intérêt de prendre en compte les caractéristiques de l'organisation temporelle du travail que sont le poste travaillé et le moment du poste observé dès lors que l'on cherche à mieux comprendre la charge de travail telle que traduite par les perceptions de l'environnement de travail chez les personnels de soins. Le recueil des perceptions des salariés directement en situation de travail, et cela en début, milieu et fin de poste, a ainsi permis 
d'appréhender les liens dynamiques qui peuvent exister entre les demandes et les ressources perçues dans un environnement de travail. En référence à la conception théorique développée par Demerouti et al. (2001), nous avons considéré ici les demandes incluant les interférences entre vie au travail et vie hors travail (Greenhaus \& Beutell, 1985), en plus des demandes psychologiques et physiques liées au travail (Theorell \& Karasek, 1996), et les ressources, correspondant à la disponibilité des ressources techniques et humaines en plus du soutien professionnel des collègues et des supérieurs.

En premier lieu, nos résultats confirment l'hypothèse de variations au cours du poste des perceptions de l'environnement de travail mais seulement pour les demandes. Plus précisément, les demandes psychologiques sont perçues dans cette population comme plus élevées en début mais aussi en milieu de poste, comparées à la fin du poste. Si l'on considère, à l'instar de Theorell et Karasek (1996), que ce sont les demandes psychologiques professionnelles qui mesurent la charge mentale de travail (mais non les demandes physiques), on peut penser que, chez les personnels de soins de l'échantillon, la charge de travail est plus élevée en début de poste comme cela a également été montré dans d'autres situations professionnelles (Cariou et al., 2008 ; Andorre \& Quéinnec, 1998 ; Mélan et al., 2007). Cependant, dans l'échantillon, cette phase de forte charge se poursuit audelà de la première heure sur toute la première moitié du poste pour ne diminuer qu'au cours de la deuxième moitié du poste. On peut supposer que si une activité de supervision accrue caractérise la première heure du poste chez des opérateurs en charge d'un processus dynamique, la planification de tâches spécifiques dans les services de soins (soins et médication, toilettes, repas,...) conduit à considérer que la charge de travail se caractérise différemment dans cette situation. Cette interprétation est renforcée par le fait que les demandes physiques auxquelles sont soumis les personnels de soins varient également en cours de poste de travail. En effet, les demandes physiques sont perçues comme plus faibles en début de poste comparé au milieu et à la fin du poste. Cette augmentation de la charge physique de travail traduit probablement un effet combiné de la fatigue accumulée tout au long du poste (Folkard \& Tucker, 2003 ; Tucker et al., 1998) et du coût engendré par le haut niveau de demandes psychologiques observées sur la première moitié du poste (Akerstedt, 2003, 2007).

Pris ensemble, ces résultats soulignent l'intérêt d'une prise en compte simultanée de ces deux types de demandes, et ce de façon nuancée à différents moments du poste. Ainsi, si les demandes psychologiques constituent un paramètre important pour décrire la charge de travail, nos résultats conduisent à considérer, dans certaines situations de travail, à la fois les demandes psychologiques et les demandes physiques pour définir la charge de travail, du moins lorsque l'on effectue une analyse détaillée sur un même poste de travail. D'autres auteurs (Devereux, Vlachonikolis, \& Buckle, 2002 ; Hämmig \& Bauer, 2013) ont déjà souligné l'importance des demandes physiques dans la perception d'un environnement de travail stressant, et cela plus particulièrement dans des métiers impliquant des efforts et des contraintes physiques importants comme c'est le cas pour l'échantillon constitué d'infirmières et d'aides soignantes, réalisant de nombreuses tâches physiques auprès des patients. 
Par ailleurs, les résultats obtenus indiquent des niveaux d'autonomie et de soutien constants en cours de poste, ne permettant pas de conclure à un environnement de travail stressant à partir de la seule analyse des effets du moment du poste. Or, les résultats relatifs aux interactions entre la phase du poste et le poste travaillé ont permis de mettre en perspective les perceptions des demandes et des ressources de l'environnement de travail. Comme nous l'avions supposé, le poste de nuit présente des caractéristiques spécifiques par rapport aux postes du matin et de l'aprèsmidi. Ce poste est le plus "délicat" au regard des connaissances sur les rythmes biologiques et leurs conséquences, la nuit, en termes de baisse de vigilance et de performance, et d'une augmentation de la somnolence (Akerstedt et al., 2004 ; Dinges et al. 1997 ; Drake et al., 2004 ; Folkard et al., 2005 ; Tepas \& Carvalhais, 1990). Cependant, en termes de perception de l'environnement de travail, les résultats suggèrent que ce poste semble le plus "confortable». Les personnels interrogés au cours de ce poste signalent en effet les niveaux les plus faibles de demandes physiques et psychologiques mais aussi les niveaux les plus élevés d'autonomie et de ressources techniques et humaines. Cette configuration correspond, dans le modèle de Theorell et Karasek (1996), à des situations de faible tension professionnelle. Néanmoins, la fin de ce poste est caractérisée par une augmentation de la charge de travail puisque, tant les demandes physiques que les demandes psychologiques sont perçues comme plus importantes une fois passé le milieu du poste alors que les ressources restent stables. Cette charge accrue par rapport au reste du poste doit refléter l'effet de la fatigue accumulée et la baisse de vigilance, deux manifestations associées au travail de nuit (les observations sont réalisées entre $5 \mathrm{~h}$ et $6 \mathrm{~h} 30$ du matin ; Cariou et al., 2008 ; Galy, Camps, \& Mélan, 2004 ; Galy, Mélan, \& Cariou, 2008 ; Garde et al., 2009 ; Keller, 2009).

Au-delà de ces facteurs, la nature de l'activité de travail et les facteurs organisationnels doivent également être pris en considération. L'importance de ces facteurs dans la compréhension de la détresse psychologique au travail a été soulignée par Marchand et Vandenberghe (2015). Dans notre étude, l'observation de demandes moindres associées à davantage de ressources paraît cohérente avec la réalité organisationnelle de la plupart des services de soins dans lesquels l'activité est ralentie la nuit (Toupin, 2005), les cadres infirmiers absents et les personnels globalement moins nombreux (organisation offrant une plus grande autonomie aux personnels présents même si cela réduit considérablement le soutien professionnel disponible).

Les perceptions recueillies lors du poste du matin indiquent, en milieu de poste, une nette augmentation à la fois des demandes perçues, du contrôle et du soutien des collègues. Si les demandes élevées traduisent une augmentation de la charge de travail, la disponibilité perçue du soutien social des collègues peut atténuer la tension induite. Le milieu de poste présente alors les caractéristiques des situations suscitant davantage de motivation ou stress positif (Karasek \& Theorell, 1990). Ces résultats concordent avec les connaissances relatives à l'activité des personnels de soins au cours de la matinée (réalisation des soins, prélèvements, préparations multiples, actes de coordination...; Estryn-Behard \& Fouillot, 
1990). Le ralentissement des activités de soin à la fin du poste pourrait expliquer la baisse des demandes psychologiques perçues. Associée à un niveau élevé de soutien social et de la disponibilité des ressources techniques et humaines, la fin de poste peut constituer un moment de moindre tension.

L'examen des résultats du poste de l'après-midi tend à soutenir l'idée qu'il s'agirait du poste pour lequel la situation est la plus "inconfortable " pour ces personnels de soins. Des niveaux de demandes physiques, élevés dès le début du poste et croissants sur la tenue du poste, et une diminution progressive des ressources (soutien social des collègues et les ressources techniques et humaines) traduisent une augmentation constante de la tension. La tension accrue traduirait ici plutôt des contraintes spécifiques comme des activités relationnelles avec les familles (Estryn-Behar \& Fouillot, 1990). Peut s'ajouter à cela la réduction du nombre de personnels présents en fin de poste, les équipes de jour ayant terminé leur poste et quitté les services tandis que les équipes de nuit n'ont pas encore rejoint les services. De plus, alors même que la vigilance des personnels est croissante en cours d'après-midi, la fatigue accumulée au cours des heures de travail et surtout depuis le lever le matin pourrait participer à l'augmentation des demandes physiques observées.

En résumé, cette étude a permis d'étendre les connaissances relatives à l'impact du poste travaillé et de la phase du poste sur la charge de travail perçue et sur le profil dynamique de cette situation de travail à des dimensions subjectives de perception de l'environnement de travail. Les résultats soutiennent l'hypothèse selon laquelle les perceptions de l'environnement de travail varient en fonction de plusieurs composantes de l'organisation du travail comme c'est le cas de la performance et de certaines fonctions psychophysiologiques sensibles à des facteurs de l'environnement et en particulier au stress (Andorre \& Quéinnec, 1998 ; Cariou et al., 2008 ; Galy et al., 2004 ; Costa, 2010). Les principales dimensions caractérisant l'environnement de travail se combinent de deux façons différentes selon le poste travaillé, et ce de manière cohérente si l'on tient compte de l'activité de travail. Cette étude montre l'intérêt de ne pas limiter l'évaluation des ressources aux seules dimensions de contrôle et de soutien social. En effet, les résultats relatifs à la dimension " ressources techniques et humaines" confirment la pertinence de prendre en considération d'autres ressources mobilisables que le contrôle et le soutien social pour mieux comprendre la modulation des effets des situations de tension (JDR, Demerouti et al., 2001 ; Bakker, van Veldhoven, \& Xanthopoulou, 2010).

Sur le plan méthodologique, cette étude a permis d'appréhender les variations des perceptions de l'environnement de travail à une faible échelle de temps, complétant ainsi l'évaluation générale des perceptions des conditions de travail, indépendamment du poste de travail et du moment du poste telle qu'elle est réalisée habituellement. Ces données plus détaillées pourraient encourager à moduler l'organisation du travail en agissant notamment sur les caractéristiques et la distribution des tâches (exigences, degré d'autonomie, etc.) et sur les caractéristiques organisationnelles comme, par exemple, les horaires ou la durée des postes (Costa, 2010 ; Härmä \& Kecklund, 2010 ; McNeely, 2005 ; Sallinen \& Kecklund, 2010). 


\section{CONCLUSION}

Nous retiendrons de cette étude, avec toute la prudence qu'impose la taille réduite de l'échantillon, l'intérêt d'une appréhension des perceptions d'un environnement de travail donné, sans pour autant pouvoir prétendre à une généralisation à d'autres situations et secteurs professionnels. Tel que le suggère Siegrist (2010), c'est auprès d'une même population ou d'une même entreprise ou organisation qu'il conviendrait d'utiliser des indicateurs subjectifs conjointement à des indicateurs objectifs de charge de travail. Des études complémentaires devraient intégrer une analyse de l'activité de travail afin de confirmer les hypothèses relatives au lien entre l'organisation des tâches au cours de la journée de 24 heures et du poste et les perceptions de la charge de travail et d'appréhender les stratégies individuelles et/ou collectives mises en place par les salariés (Cazabat et al., 2008 ; Farmer et al., 2005). Ces préconisations méthodologiques permettraient également de tester l'hypothèse d'un lien direct avec les contraintes liées à la rythmicité circadienne de l'état fonctionnel des personnels concernés (Ackerstedt et al., 2004 ; Galy \& Gaudin, 2014). De même, il serait pertinent d'inclure d'autres facteurs socio-biographiques susceptibles d'interférer avec la perception de l'environnement de travail, en particulier l'âge et la fonction des salariés en lien notamment avec les demandes physiques du travail (Boudreau et al., 2013 ; Costa, 1995, 2010 ; Griffiths et al., 2011 ; Hämmig \& Bauer, 2013 ; Keller, 2009 ; Reinke et al., 2015).

\section{BIBLIOGRAPHIE}

Akerstedt, T. (2003). Shift work and disturbed sleep/wakefulness. Occupational Medicine, 53, 89-94.

Akerstedt T. (2007). Altered sleep/wake patterns and mental performance. Physiology and Behavior, 90, 209-218.

Akerstedt, T., Knutsson, A., Westerholm, P., Theorell, T., Alfredsson, L., \& Keclund, G. J. (2004). Mental fatigue, work and sleep. Fournal of Psychosomatic Research, 57, 427-433.

Andorre, V., \& Quéinnec,Y. (1998). Changes in supervisory activity of a continuous proc during night and day shifts. International fournal of Industrial Ergonomics, 21, 179-186.

Bakker, A.B., van Veldhoven, M., \& Xanthopoulou, D. (2010). Beyond the DemandControl Model. Thriving on High Job Demands and Resources. Fournal of Personnel Psychology, 9(1), 3-16.

Bakker, A.B., Demerouti, E., \& Schaufeli, W.B. (2003). Dual processes at work in a call centre: an application of the Job Demands-Resources model. European fournal of Work and Organizational Psychology, 12, 393-417.

Bakker, A.B., Demerouti, E., \& Sanz-Vergel, A.I. (2014). Burnout and work engagement: the JD-R approach. Annual Review of Organizational Psychology and Organizational Behavior, 1(1), 389-411.

Barthe, B., Quéinnec, Y., \& Verdier, F. (2004). L'analyse de l'activité de travail en postes de nuit : Bilan de 25 ans de recherches et perspectives. Le Travail Humain, 67, 41-61.

Bostock, S., \& Steptoe, A. (2013). Influences of early shift work on the diurnal cortisol rhythm, mood and sleep: Within-subject variation in male airline pilots. Psychoneuroendocrinology, 38, 533-541. 
Boudreau, Ph., Dumont, G.A., \& Boivin, D.B. (2013). Circadian Adaptation to Night Shift Work Influences Sleep, Performance, Mood and the Autonomic Modulation of the Heart. Plos One, 8(7) doi:10.1371/journal.pone.0070813

Cariou, M., Galy, E., \& Mélan, C. (2008). Differential 24-h variations of alertness and subjective tension in process controllers: investigation of a relationship with body temperature and heart rate. Chronobiology International, 25, 597-609.

Cazabat, S., Barthe, B., \& Cascino, N. (2008). Charge de travail et stress professionnel : deux facettes d'une même réalité ? Etude exploratoire dans un service de gérontologie. Perspectives interdisciplinaires sur le travail et la santé [En ligne], 10-1. URL : http://pistes.revues.org/2159.

Costa, G. (1995). Occupational Stress and Stress Prevention in Air Traffic Control, Geneva, Switzerland: Conditions of Work and Welfare, International Labor Office.

Costa, G. (2010). Shift work and health: current problems and preventive actions. Safety and health at work, 1(2), 112-123.

Costa, G., Anelli, M.M., Castellini, G., Fustinoni, S., \& Neri, L. (2014). Stress and sleep in nurses employed in " $3 \times 8$ " and " $2 \times 12$ " fast rotating shift schedules. Chronobiology International, 31(10), 1169-1178.

Demerouti, E., Bakker, A.B., Nachreiner, F., \& Schaufeli, W.B. (2001). The job demands-resources model of burnout. Fournal of Applied Psychology, 86(3), 499-512.

Demerouti, E., Geurts, S., Bakker A.B., \& Euwena, M. (2004). The impact of shiftwork on work-home conflict, job attitude and health, Ergonomics, 47(9), 9871002.

Devereux, J., Vlachonikolis, I., \& Buckle, P. (2002). Epidemiological study to investigate potential interaction between physical and psychosocial factors at work that may increase the risk of symptoms of musculoskeletal disorder of the neck and upper limb. Occupational and Environmental Medicine, 59(4), 269-277.

Dinges, D.F., Pack, F., Williams, K., Gillen, K.A., Powell, J.W., Ott, G.E., Aptowicz, C., \& Pack, Al. (1997). Cumulative sleepiness, mood disturbance, and psychomotor vigilance performance decrements during a week of sleep restricted to 4-5 hours per night, Sleep. 20, 267-277.

Drake, C.L., Roehrs, T., Richardson, G., Walsh, J.K., \& Roth, T. (2004). Shift work sleep disorder: prevalence and consequences beyond that of symptomatic day workers. Sleep, 27(8), 1453-1462.

Estryn-Behar, M., \& Fouillot, J.P. (1990). Etude de la charge mentale et approche de la charge psychique du personnel soignant : analyse du travail des infirmières et aides-soignantes dans 10 services de soins. Documents pour le médecin du travail, 42(6), 131-144.

Estryn-Behar, M., \& Van der Hejden, B.I.J.M. (2012). Effects of extended work shifts on employee fatigue, health, satisfaction, work/family balance, and patient safety. Work, 41(0), 4283-4290.

Farmer, J., West, C., Whyte, B., \& Maclean, M. (2005). Primary health-care teams as adaptive organizations: exploring and explaining work variation using case studies in rural and urban Scotland. Health Services Management Research, 18, 151-164.

Folkard, S., \& Tucker, P. (2003). Shiftwork, safety and productivity. Occupational Medicine, 53, 95-101.

Folkard, S., Lombardi, D.A., \& Tucker, P.T. (2005). Shiftwork: Safety, Sleepiness and Sleep. Industrial Health, 43, 20-23.

Gadbois, C. (1975). L'analyse des emprises réciproques de la vie de travail et de la vie hors travail. Bulletin du CERP, 23(2), 117-151.

Gadbois, C. (1980). Les exigences du travail hospitalier de nuit comme facteurs de la charge de travail. Le Travail Humain, 43 (1), 17-31. 
Gadbois, C., \& Quéinnec, Y. (1984) Travail de nuit, rythmes circadiens et régulations des activités. Le Travail Humain, 47(3), 195-225.

Gadbois, C. (2004). Les discordances psychosociales des horaires postés : questions en suspens. Le Travail Humain, 67, 63-85.

Galy, E., Camps, J.F., \& Mélan, C. (2004). Performances de mémorisation en fonction de la modalité d'encodage au cours des 24 heures chez des travailleurs postés. Le Travail Humain, 67, 21-40.

Galy, E., Mélan, C., \& Cariou, M. (2008). Investigation of task performance variations according to task requirements and alertness across the 24-h day in shiftworkers. Ergonomics, 51, 1338-1351.

Galy, E., \& Gaudin, C. (2014). Vigilance et tension : effet de l'organisation du travail et de la perception de la situation de travail. Le Travail Humain, 77(4), 301-323.

Garde, A.E., Hansen, A.M., \& Hansen, J (2009). Sleep length and quality, sleepiness and urinary melatonin among healthy Danish nurses with shift work during work and leisure time. International Archives of Occupational and Environmental Health, 82, 1219-1228.

Greenhaus, J.H., \& Beutell, N.J. (1985). Sources and conflict between work and family roles. Academy of Management Review, 10(1), 76-88.

Griffiths, K.L., Mackey, M.G., \& Adamson, B.J. (2011). Behavioral and Psychophysiological Responses to Job Demands and Association with Musculoskeletal Symptoms in Computer Work. Fournal of Occupational Rehabilitation, 21(4), 482-492. doi: 10.1007/s10926-010-9263-3.

Hämmig, O., \& Bauer, G.F. (2013). The social gradient in work and health: a cross-sectional study exploring the relationship between working conditions and health inequalities. BMC Public Health, 13(1), 1170.

Härmä, M., \& Kecklund, G. (2010). Shift work and health - how to proceed? Scandinavian Fournal of Work Environment and Health, 36(2), 81-84.

Hart, S.G., \& Staveland, L.E. (1988). Development of NASA-TLX (Task Load Index): Results of empirical and theoretical research. In P.A. Hancock, \& N. Meshkati (Eds.), Human mental workload (pp.139-193). Oxford, England: North-Holland. doi:10.1016/S0166-4115(08)62386-9.

Johnston, D.W., Beedie, A., \& Jones, M.C. (2006). Using computerised ambulatory diaries for the assessment of job characteristics and work-related stress in nurses. Work \& Stress, 20, 163-172.

Kang, J.H., Miao, N.F., Tseng, I.J., Sithole, T., \& Chung, M.H. (2015). Circadian Activity Rhythms and Sleep in Nurses Working Fixed 8-hr Shifts. Biological Research for Nursing, 17(3), 348-355.

Karasek, R. (1979). Job demand, job decision latitude, and mental strain: Implications for redesign. Administrative Science Quaterly, 24, 285-307.

Karasek, R., \& Theorell, T. (1990) - Healthy Work: Stress, Productivity, and the Reconstruction of Working Life. - New York: Basic Books.

Karasek, R., Brisson, C., Kawakami, N., Houtman, I., Bongers, P., \& Amick, B. (1998). The Job Content Questionnaire (JCQ): An instrument for internationally comparative assessments of psychosocial job characteristics. Fournal of Occupational Health Psychology, 3(4), 322-355.

Keller, S.M. (2009). Effects of extended work shifts and shift work on patient safety, productivity, and employee health. Official fournal of the American Association of Occupational Health Nurses, 57(12), 497-502.

Marchand, C., \& Vandenberghe, C. (2015). Soutien organisationnel perçu, perception de pertes de ressources, et santé psychologique : l'effet modérateur de l'affectivité négative. Travail Humain, 78(3), 193-216.

McClelland, L.E., Switzer, F.S., \& Pilcher, J.J. (2013). Changes in decision-making in nurses across a 12-hour day shift. Occupational Medicine, 63(1), 60-65. doi: $10.1093 /$ occmed/kqs 189 . 
McNeely, E. (2005). The consequences of job stress for nurses' health: time for a check-up. Nursing Outlook, 53, 291-299.

Mélan, C., Cascino N., \& Galy E. (2014). Within-shift Investigation of Fob Perception, cognitive Performance, Alertness and their Relationship with ATC Operations. Proceedings of the 31st Conference of the European Association of Aviation Psychology, Malte.

Mélan, C., \& Cascino, N. (2014). A multidisciplinary approach of workload assessment in real-job situations: Investigation in the field of aerospace activities. Frontiers in Psychology, Quantitative Psychology and Measurement, 5: 964. doi 1003389/fpsyg.2014.00964.

Mélan, C., Cascino, N., Galy, E., \& Barthe, B. (2012). Mesurer la charge de travail : une approche pluridisciplinaire in C. Courtet, \& M. Gollac (Eds.), Risques du travail, la santé négociée (pp. 189-204). Paris: La Découverte.

Mélan, C., Galy, E., \& Cariou, M. (2007). Mnemonic Processing in Air Traffic Controllers (ATCs): Effects of Task Parameters and Work Organization. International fournal of Aviation Psychology, 17, 391-409.

Nicoletti, C., Müller, C., Hayashi, C., Nakaseko, M., Tobita I., \& Läubli, T. (2015). Circadian rhythm of heart rate and physical activity in nurses during day and night shifts. European fournal of Applied Physiology, 115(6), 1313-1320.

Ota, A., Masueb, T., Yasudaa, N., Tsutsumic, A, Minod, Y., \& Oharaa, H. (2005). Association between psychosocial job characteristics and insomnia: an investigation using two relevant job stress models-the demand-control-support (DCS) model and the effort-reward imbalance (ERI) model. Sleep Medicine, 6, 353-358.

Peplonska, B., Bukowska, A., \& Sobala, W. (2014). Rotating night shift work and physical activity of nurses and midwives in the cross-sectional study in Łódź, Poland. Chronobiology International, 31(10), 1152-1159.

Rathore, H., Shukka, K., Singh, S., \& Tiwari, G. (2012). Shift work - problems and its impact on female nurses in Udaipur, Rajasthan India. Work, 41(0), 43024314.

Reinke, L., Özbay, Y., Dieperink, W., \& Tulleken, J.E. (2015). The effect of chronotype on sleepiness, fatigue, and psychomotor vigilance of ICU nurses during the night shift. Intensive Care Medicine, 41(4), 657-466.

Rutenfranz, J., Colquhoun, W.P., Knauth, P., \& Ghata, J.N. (1977). Biomedical and psychosocial aspects of shift work. Scandinavian Fournal of Work, Environment and Health, 3(4), 165-182.

Sallinen, M., \& Kecklund, G. (2010). Shift work, sleep and sleepiness - differences between shift schedules and systems. Scandinavian fournal of Work, Environment and Health, 36(2), 121-133.

Schaefer, E.W., Williams, M.V., \& Zee, P.C (2012). Sleep and circadian misalignment for the hospitalist: a review. Fournal of Hospital Medicine, 7(6), 489-496.

Shaufeli, W.B., \& Bakker, A.B. (2004). Job demands, job resources, and their relationship with burnout and engagement: A mulyti-sample study. Fournal of Organizational Behavior, 25, 293-315.

Siegrist, J. (2010). Effort-reward imbalance at work and cardiovascular diseases. International Fournal of Occupational Medicine and Environmental Health, 23, 279-285.

Teasley, S.L., Sexton, K.A., Carroll, C.A., Cox, K.S., Riley, M., \& Ferriell, K. (2007). Improving Work Environment Perceptions for Nurses Employed in a Rural Setting. The fournal of Rural Health, 23(2), 179-183.

Tepas, D.I., \& Carvalhais, A.B. (1990). Sleep patterns of shiftworkers. Fournal of Occupational Medicine, 5, 199-208.

Theorell, T., \& Karasek R. (1996). Current issues relating to psychosocial job strain and cardiovascular disease research. Fournal of Occupational Health Psychology, 1(1), 9-26. 
Toupin, C. (2005). L'élaboration des stratégies de travail nocturnes : le cas des infirmières de nuit d'un service de pneumologie français. Perspectives Interdisciplinaires sur le travail et la santé [En ligne], 7-1. http://pistes.revues. org/3253.

Tucker, P., Barton, J., \& Folkard, S. (1996). Comparison of eight and 12 hour shifts: impacts on health, wellbeing, and alertness during the shift. Occupational Environment Medicine, 53(11), 767-772.

Tucker, P., Smith, L., Macdonald, I., \& Folkard, S. (1998). The impact of early and late shift changeovers on sleep, health, and well-being in 8- and 12-hour shift systems. Fournal of Occupational Health Psychology, 3(3), 265-275.

Van Emmerik, I.J.H., Bakker, A.B., \& Euwema, M. C. (2009). Explaining employees' evaluations of organizational change with the job-demands resources model. Career Development International, 14(6), 594-613.

Conflit d'intérêt : aucun

Manuscrit reçu : juillet 2014.

Accepté après révision par F. Darses : octobre 2015. 


\section{Annexe 1 : Questionnaire d'évaluation des perceptions de l'environnement de travail}

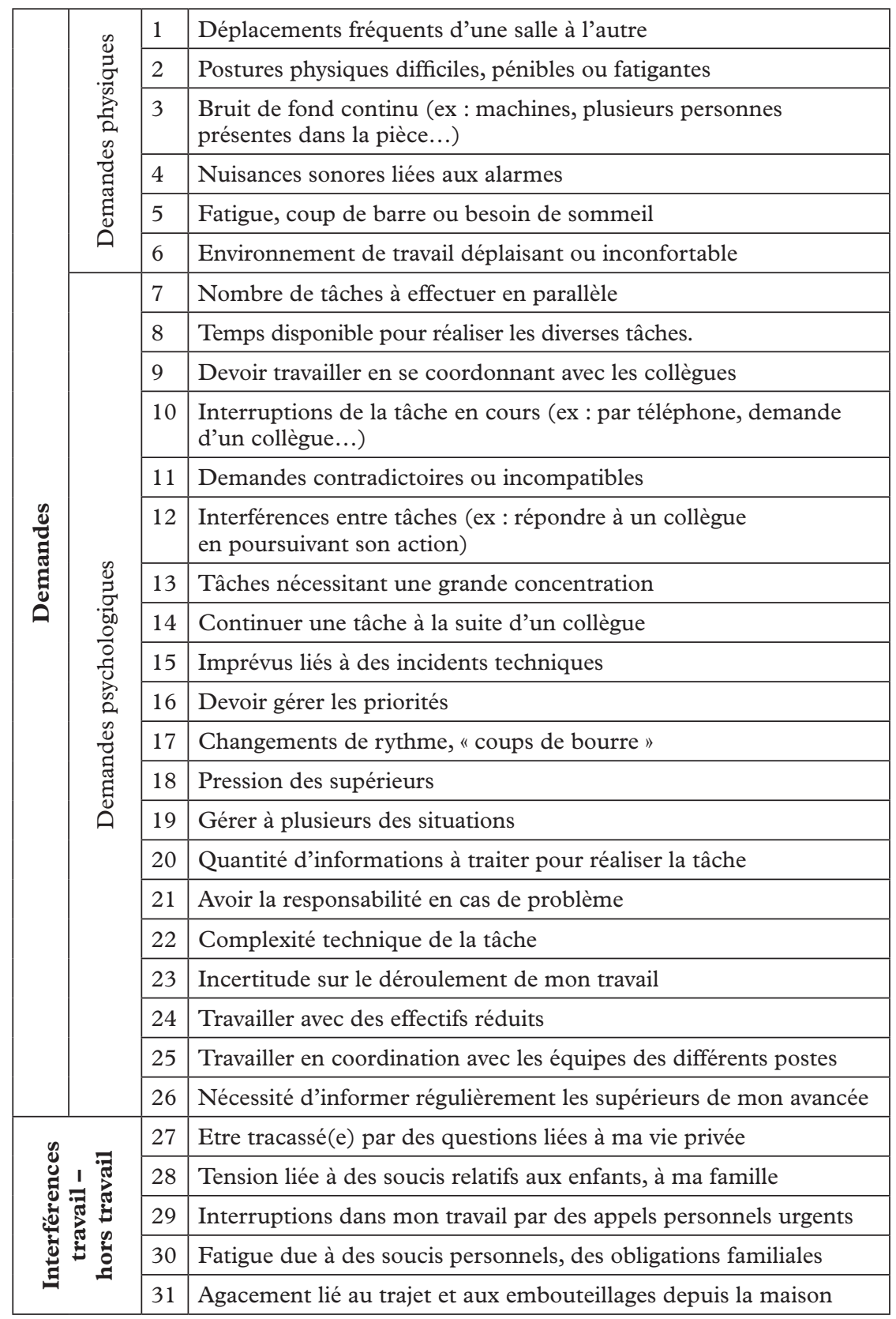




\begin{tabular}{|c|c|c|c|}
\hline \multirow{13}{*}{$\begin{array}{l}\stackrel{0}{0} \\
\stackrel{0}{\Xi} \\
\stackrel{\Xi}{0}\end{array}$} & \multirow{5}{*}{ 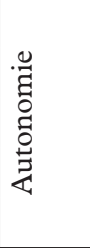 } & 32 & Liberté dans la manière de réaliser mon travail \\
\hline & & 33 & Autonomie pour prendre des décisions ponctuelles \\
\hline & & 34 & Dépendre du travail d'autrui pour avancer dans mon propre travail \\
\hline & & 35 & Occasion de recourir à mon initiative personnelle, mon jugement \\
\hline & & 36 & Participation aux prises de décisions \\
\hline & \multirow{8}{*}{ 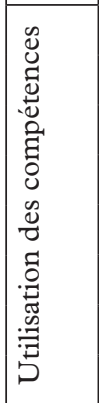 } & 37 & Clarté des tâches à réaliser \\
\hline & & 38 & Précision des procédures relatives aux différentes situations \\
\hline & & 39 & Diversité des compétences sollicitées \\
\hline & & 40 & Simplicité des tâches à réaliser \\
\hline & & 41 & Apprendre des choses nouvelles \\
\hline & & 42 & Diversité des tâches \\
\hline & & 43 & Opportunités de développer des aptitudes nouvelles \\
\hline & & 44 & Possibilités d'utiliser toutes mes compétences \\
\hline \multirow{7}{*}{ 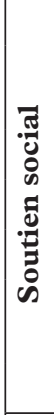 } & SUP & 45 & Ecoute de la part du supérieur \\
\hline & COL & 46 & $\begin{array}{l}\text { Possibilité de se faire remplacer par un collègue (ex : pour aller au } \\
\text { C.E...) }\end{array}$ \\
\hline & COL & 47 & Ambiance tendue entre collègues \\
\hline & SUP & 48 & Conseils et idées des supérieurs pour résoudre des problèmes \\
\hline & COL & 49 & Ecoute de la part des collègues \\
\hline & COL & 50 & $\begin{array}{l}\text { Aide ponctuelle de collègues sur un problème donné (ex : conseils, } \\
\text { idées...) }\end{array}$ \\
\hline & SUP & 51 & Climat tendu avec les supérieurs \\
\hline \multirow{5}{*}{\multicolumn{2}{|c|}{ 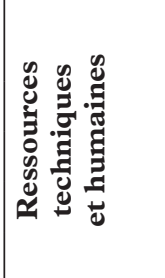 }} & 52 & $\begin{array}{l}\text { Temps et lieu d'échanges entre collègues (niveau personnel } \\
\text { et relationnel) }\end{array}$ \\
\hline & & 53 & Possibilité de coordination entre les membres de l'équipe \\
\hline & & 54 & Equipements adaptés pour faire correctement mon travail \\
\hline & & 55 & Disponibilité des informations nécessaires pour faire mon travail \\
\hline & & 56 & Coordination entre les équipes assurant les différents postes \\
\hline
\end{tabular}




\section{Annexe 2 : Consignes}

\section{Consigne temporellement située : début de poste}

Identifiant :

Vous êtes à votre poste de travail depuis maintenant environ 1 heure. Voici une liste de propositions correspondant aux différentes caractéristiques possibles dans des situations de travail. Pour chacun d'eux, pouvezvous indiquer dans quelle mesure ceux-ci ont été présents durant cette première heure dans la réalisation de votre travail ? Pour cela, veuillez entourer le chiffre correspondant le mieux à votre sentiment personnel sachant que 1 correspond à "pas présent du tout " et 6 "très souvent présent ».

\section{Consigne temporellement située : milieu de poste}

\section{Identifiant :}

Heure :

Vous êtes maintenant à peu près à la moitié de votre journée de travail. Voici une liste de propositions correspondant aux différentes caractéristiques possibles dans des situations de travail. Pour chacun d'eux, pouvez-vous indiquer dans quelle mesure ceux-ci ont été présents durant cette première heure dans la réalisation de votre travail ? Pour cela, veuillez entourer le chiffre correspondant le mieux à votre sentiment personnel sachant que 1 correspond à "pas présent du tout " et 6 "très souvent présent ».

\section{Consigne temporellement située : fin de poste}

Identifiant :

Heure :

D'ici environ 1 heure vous aurez terminé votre journée de travail. Voici une liste de propositions correspondant aux différentes caractéristiques possibles dans des situations de travail. Pour chacun d'eux, pouvez-vous indiquer dans quelle mesure ceux-ci ont été présents durant cette première heure dans la réalisation de votre travail ? Pour cela, veuillez entourer le chiffre correspondant le mieux à votre sentiment personnel sachant que 1 correspond à "pas présent du tout " et 6 "très souvent présent ».

\begin{tabular}{|l|llllllll|}
\hline & \multicolumn{3}{|l|}{$\begin{array}{l}\text { Pas présent } \\
\text { du tout }\end{array}$} & \multicolumn{3}{l|}{$\begin{array}{l}\text { Très souvent } \\
\text { présent }\end{array}$} \\
\hline Déplacements fréquents d'une salle à l'autre & 1 & 2 & 3 & 4 & 5 & 6 \\
\hline $\begin{array}{l}\text { Postures physiques difficiles, pénibles } \\
\text { ou fatigantes }\end{array}$ & 1 & 2 & 3 & 4 & 5 & 6 \\
\hline$(\ldots)$ & 1 & 2 & 3 & 4 & 5 & 6 \\
\hline
\end{tabular}


